\title{
Study of Heat Engineering Homogeneity Fragments of Enclosing Structures in the Climatic Chamber
}

\author{
Gregory P. Vasilyev ${ }^{1}$, Vitaly A. Leskov², Victor F. Gornov², Marina V. Kolesova ${ }^{2}$, Vladimir A. Lichman ${ }^{2}$ \\ ${ }^{1}$ JSC «INSOLAR-INVEST», Moscow, Russia, 121433, Moscow, Filevskaya Street, house 32, building 3 \\ ${ }^{2}$ Vitaly A. Leskov, Victor F. Gornov, Marina V. Kolesova, Vladimir A. Lichman \\ JSC «INSOLAR-INVEST», Moscow, Russia, 121433, Moscow, Filevskaya Street, house 32, building 3
}

\begin{abstract}
The article describes a method of experimental determination of the coefficient of heat engineering homogeneity of enclosing structures buildings used during thermal testing in a climate chamber. The method is based on obtaining a matrix of temperature distribution on the inner surface of a fragment of enclosing structure. The matrix is formed by collecting data from a thermovision controller and data received from the temperature sensors installed at selected points.
\end{abstract}

\section{Introduction}

One of the priority tasks to be solved by increasing the energy efficiency of buildings is to increase the heatshielding properties of external enclosing structures. Heterogeneous sections of the outer enclosing structure, the so-called "cold bridges" - joints, dowels, thermofiller etc., occupy a large part of the area of the building of fences, so they significantly affect the shielding properties and durability of the building. Search technical solutions aimed at improving the uniformity of heat engineering exterior enclosing structure is one of on the front burner problems solving in the modern Thermal Engineering. Today is quite well developed as a simplified and detailed methods of calculation to determine the transport coefficients (thermal resistance) in walling [1-4].

This paper presents the experimental method of determining the coefficient of thermal homogeneity of enclosing structures used to conduct thermal testing in the climate chamber, in which are stationary heat transfer conditions [5,6]. The method is based on obtaining the matrix of the temperature distribution $\tau_{\text {int }}(x, y)$ on the inner surface of a fragment of enclosing structure by using a thermovision controller. Thermovision control of a surface of enclosing structures is carried out according to GOST R 548522011 [7]. However, the thermograms obtained due to the influence of various random effects are qualitative in nature. In the program processing of thermograms has several options that allow you to change their temperature and color match. The main parameters include the emissivity the coefficient of a surface of a construction, - measure the radiating emitted by an object, compared to the full radiator at the same temperature and see the reflection of temperature parameter used to compensate for radiation surrounding bodies. The proposed method is based on the fact that the field of temperature distribution obtained thermal imaging method, it is possible to adjust (to hold a "calibrated thermal images") with the temperature values measured by sensors installed in some specially selected points. Thus, high-quality thermogram is converted into a digital array of quantitative data of temperature distribution on the inner surface of the enclosing structure.

In world practice, this question has been studied and documented in the international standards EN ISO 6946, ISO 14683: 2007, EN ISO 13370, ISO 10211: 2007 , ISO 10211, etc. the provisions of these standards were examined by Russian specialists. On their basis taking into account domestic experience is designed as a simplified and detailed methods for determination of transfer coefficients (thermal resistances) enclosing structures of buildings and is included in the Russian standards GOST R 54852-2011, GOST 26602.1-99 and GOST R 54853-2011, which contains the methodology of laboratory tests of the fragment of walling in climate chamber and method of processing the results.

This article describes an experimental method of determining the coefficient of heat engineering uniformity of the building envelope which is being used to conduct thermal testing in the climate chamber, in which are stationary conditions of heat exchange [6], which allows to significantly reduce the complexity 
and therefore the cost of testing in comparison with methods described in GOST R 54853-2011.

This regulatory document includes a method comprising the use of an environmental chamber providing a temperature difference in the sample and which consists in that at the preparatory stage of testing determine the approximate temperature distribution and heat flux on the surface of the sample, using, as an auxiliary tool, thermal imaging camera, based on what design the layout of multiple temperature sensors and calorimeter. Then the sample in a climatic chamber output to the stationary thermal regime at a given temperature difference and take readings of the temperature sensors and calorimeter to determine the temperature fields and heat fluxes taking into account the heterogeneity of heat transfer resistance of the test specimen.

The disadvantage of the above method is the considerable complexity and length associated with the development of the layout and directly installing a large number of sensors.

The proposed method aims at reducing the complexity of experimental determination of coefficient of heat engineering uniformity of enclosing structures of buildings and based on obtaining the matrix of the temperature distribution on the inner surface of a fragment of enclosing structure by using a thermal imaging controller. Thermal imaging control of a surface of enclosing structures is carried out according to GOST R 54852-2011. [7] However, the thermograms obtained due to the influence of various random effects are qualitative in nature. In the program processing of thermograms has several options that allow you to change their temperature and color match. The main parameters include the emissivity the coefficient of a surface of a construction, - measure the radiating emitted by an object, compared to the full radiator at the same temperature and see the reflection of temperature - parameter used to compensate for radiation surrounding bodies. The proposed method is based on the fact that the field of temperature distribution obtained thermal imaging method, it is possible to adjust (to hold a "calibrated thermal images") with the temperature values measured by sensors installed in some specially selected points. Thus, high-quality thermogram is converted into a digital array of quantitative data of temperature distribution on the inner surface of the enclosing structure.

\section{Defining of the coefficient of the thermotechnical homogeneity of a fragment of an outer enclosing structure}

In the first stage, using data of thermography, define thermal homogeneous areas of a fragment of an outer enclosing structure. Further by a contact method with temperature sensors temperature measured at selected points on the inner surface of thermal homogeneous areas of a fragment of an enclosing structure. Thermograms of fragments of the inner surface of an enclosing structure which are under the testing is processed using special programs. These programs are developed by the manufacturer of thermal imaging equipment (for example, the program ThermaCAM, FLIR systems). With the above parameters make "calibrating thermogram" on the temperature measured by the sensors at selected points, i.e. at selected points achieve coincidence of temperatures measured by the contact method and obtained by thermography. Next thermograms are digitized and processed by the developed in MATLAB program. The basic relation of algorithm of processing of thermograms following. Heat flow density $q_{0} \cdot \mathrm{W} / \mathrm{m}^{2}$, passing through the thermotechnical homogeneity the plot of a fragment of the inner surface of an enclosing structure area of $A_{0}$ : $q_{0}=\alpha_{\text {int }} \iint_{A_{0}}\left(t_{\text {int }}-\tau_{\text {int }, 0}(x, y)\right) d x d y$

Heat flow density $q_{r} \cdot W / \mathrm{m}^{2}$, passing through the entire plot of a fragment of the inner surface of of an enclosing structure area of $A_{i}, \mathrm{~m}^{2}$ :

$q_{r}=\alpha_{\text {int }} \iint_{A_{i}}\left(t_{\text {int }}-\tau_{\text {int }}(x, y)\right) d x d y$

$t_{\text {int }},{ }^{o} \mathrm{C}$ - air temperature of a heat compartment of a climatic chamber; $\tau_{\text {int }, 0}(x, y),{ }^{\circ} \mathrm{C}$-values of temperature on a thermal homogeneous plot of a fragment enclosing structure of a sample; $\tau_{\text {int }}(x, y),{ }^{\circ} \mathrm{C}$ - a matrix of values of temperatures on the entire inner surface of a fragment of an enclosing structure of a sample; $\alpha_{\text {int }}\left(\mathrm{W} / \mathrm{m}^{2} \cdot K\right)$ the coefficient of heat transfer of inner surface of a fragment of an enclosing structure of a sample.

Assuming that the heat transfer coefficient is constant for a given the fragment of an enclosing structure of the sample, calculate the coefficient of thermal homogeneity of a fragment of an enclosing structure

$r_{i}=\frac{\iint_{0}\left(t_{\text {int }}-\tau_{\text {int }, 0}(x, y)\right) d x d y}{\iint_{A_{i}}\left(t_{\text {int }}-\tau_{\text {int }}(x, y)\right) d x d y}$ 
Or, averaging values of temperatures around the fragment $\tau_{c p}$ and homogeneity thermotechnical portion $\tau_{c p, 0}$, we obtain

$r_{i}=\frac{t_{\text {int }}-\tau_{c p, 0}}{t_{\text {int }}-\tau_{c p}}$

The coefficient of thermotechnical homogeneity of the entire sample of the outer enclosing structure is calculated as a weighted average of the fragments:

$$
r=\sum A_{i} r_{i} / \sum A_{i}
$$

If at the selected points of the fragment enclosing structure in addition to the temperature sensor installed a heat flowmeter and measure heat flow, then it will determine the coefficient of heat transfer of the inner surface $\alpha_{\mathrm{int}}\left(\mathrm{W} / \mathrm{m}^{2} \cdot K\right)$, calculated from equation (2) heat flow $q_{r} \cdot W / \mathrm{m}^{2}$, and further will determine the value of thermaresistance of a fragment of enclosing structure:

$$
R_{r, i}=\frac{\left(t_{\text {int }}-t_{\text {ext }}\right)}{q_{r}}
$$

where $t_{\text {ext }},{ }^{o} \mathrm{C}$ - measured temperature of the air in the cold compartment of the climate chamber.

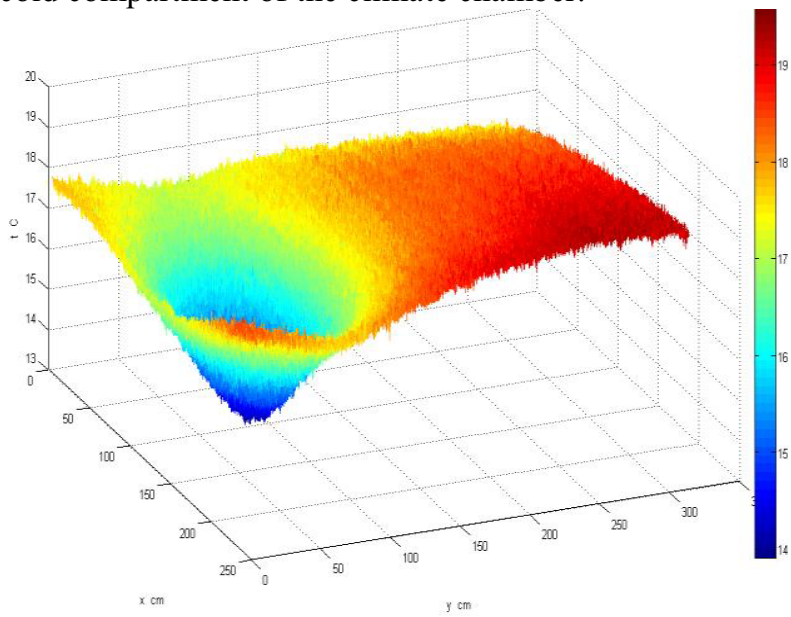

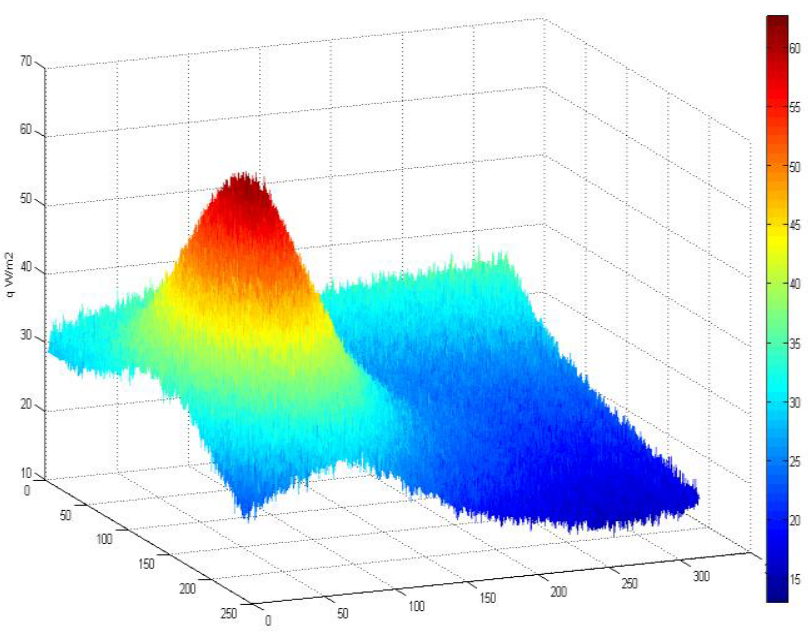

Figure 1. Distribution of temperature $\tau_{\text {int }}(x, y)$ (above) and the density of heat flow $q_{r}(x, y)$ (bottom) on the inner surface of the fragment of the inner surface of the three-layer reinforced concrete panel. The size of the temperature matrix $\tau_{\text {int }}(x, y)$ is 320 on 240 pixels. The coefficient of thermotechnical homogeneity of the fragment is $r_{i}=0,67$.

Fig. 1 shows the results of processing the images is three-dimensional graphs of the distributions of temperature $\tau_{\text {int }}(x, y)$ and heat flow density $q_{r}(x, y)$ of the fragment of the inner surface of three-layer reinforced concrete panels. The size of the temperatures matrix $\tau_{\text {int }}(x, y)$ of the inner surface is 320 on 240 pixels, the size of the fragment is $A_{i}=0,9 \cdot \mathrm{m}^{2}$. The average value of temperature of the thermotechnical homogeneity area $\tau_{c p, 0}=18,2 \cdot{ }^{0} \mathrm{C}$; The average value of temperature of the whole fragment $\tau_{c p}=16,8 \cdot{ }^{0} C$; the air temperature in the warm compartment of a climate chamber near this fragment; in the cold compartment $t_{\mathrm{ext}}=-27,2 \cdot{ }^{0} \mathrm{C}$; the value of the coefficient of thermotechnical homogeneity of the given fragment $r_{i}=0,67$; heat transfer resistance. The value of the coefficient of thermotechnical homogeneity of the given fragment $r_{i}=0,67$; the thermal resistance $R_{r, i}=2,21 \cdot m^{2} \mathrm{~K} / \mathrm{W}$. 

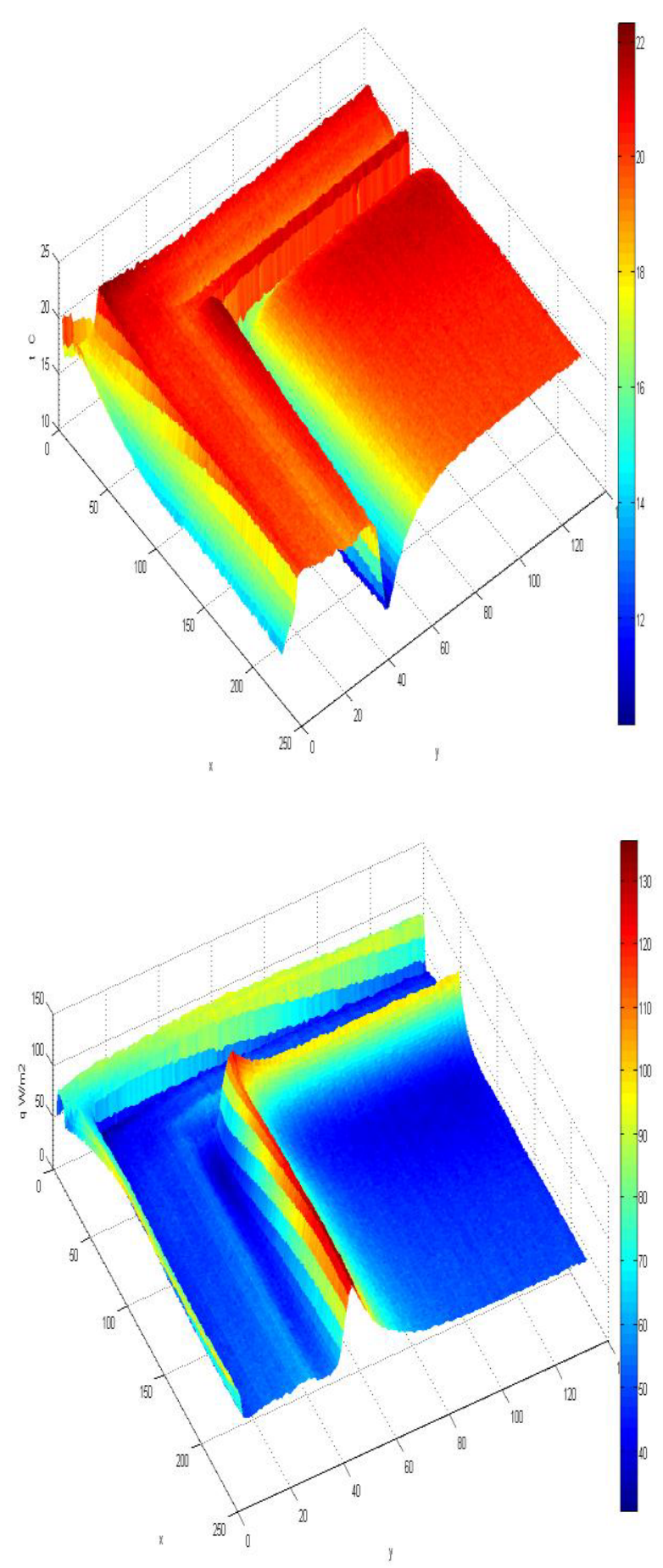

Figure 2. Distribution of the temperature $\tau_{\text {int }}(x, y),{ }^{0} C$ (above) and of the heat flow density $q_{r}(x, y), W / m^{2}$ (below) on the surface of an element of the internal surface of a window.

As for the second example - Figure 2 shows the distribution of the temperature $\tau_{\text {int }}(x, y)$ and of the heat flow density $q_{r}(x, y)$ of an element of the surface of a window (the glazing and the frame), obtained in a climatic chamber. Thermal resistance of this window unit element is $R_{r}=(21-(-25)) / 57=0,81 \cdot\left(m^{2} \cdot K\right) / W$.

\section{Conclusions}

The described method of determining the coefficient of the thermotechnical homogeneity and the thermal resistance of testing fragments of enclosing structures in a climate chamber. It is based on the application as the main research tool thermal imaging cameras, followed by calibrating the results according to the testimony of one or more temperature sensors or calorimeter.

The method can serve as complement to the methods described in standards [5, 6], significantly (several times) reduces the time and cost of testing.

\section{Acknowledgments}

The Research was conducted by JSC "INSOLARINVEST" with the financial support of the Ministry of Education and Science of Russia. Unique identifier of the project RFMEFI57614X0034.

\section{References}

1 EN ISO 6946:2007 Building components and building elements - Thermal resistance and thermal transmittance - Calculation method.

2 ISO 14683:2007 Thermal bridges in building construction - Linear thermal transmittance Simplified methods and default values.

3 ISO 10211:2007 Thermal bridges in building construction - Heat flows and surface temperatures - Detailed calculation.

4 SP 50.13330.2012 Thermal performance of the buildings.

5 GOST R 54853-2011 Bulidings and structures. Method for determination of thermal resistance and thermal coefficient of enclosing structures wiht assistance of heat flow meter.

6 GOST 26602.1-99 Windows and doors. Methods of determination of resistance of thermal transmission.

7 GOST R 54852-2011 - Buildings and structures. Method of thermovision control of enclosing structures thermal insulation quality. 